\title{
A model for minimizing feedback-length between activities of a project
}

\author{
Jafar Gheidar-Kheljani*, Sheida Shahabadi** and Seyyed Hasan Ghodsypour*** \\ * Associate Professor, Management and Industrial Engineering Department, Malek-Ashtar University of Technology, Tehran, Iran. \\ ** M.Sc. Industrial Engineering and Management Systems Department, Amirkabir University of Technology, Tehran, Iran. \\ *** Professor, Industrial Engineering and Management Systems Department, Amirkabir University of Technology, Tehran, Iran. \\ *Corresponding Author: kheljani@mut.ac.ir
}

Submitted :06/04/2020

Revised :04/05/2021

Accepted : 11/05/2021

\begin{abstract}
Proper sequencing between activities has always been one of the major issues in project management. Sometimes it is needed to redo some activities especially in product development projects. It is assumed that one of the reasons for reworking some activities and backward can be due to the incompleteness of the information needed when doing them, and it is also assumed that having complete information requires cost. In this article, a network of activities is considered, which may require a duplication of the process due to the interdependence of information between them. In this paper, using the design structure matrix concept and interdependence between activities, a twopurpose model for determining the proper sequence of activities has been provided to minimize the length of feedback and reduce the cost of completing the information using matrix concept. To accurately solve this model, the combination of Lexicographic and Augmented Epsilon Constraints is used to obtain the Pareto National Front, which shows an increase in the cost of reducing the degree of dependence between activities for reducing the feedback length between activities. From the Pareto front, the best answer was chosen in a way that, based on the economic justification of the project, the percentage of reduction in the feedback length would be higher than the percentage increase.
\end{abstract}

Keywords: Design structure matrix; Degree of information dependence; Feedback length; Lexicographic; Pareto front.

\section{INTRODUCTION}

In planning a project, some activities require rework and even remove and carry out new activities. This has led to some cases of backwardness or loop in the design of a network of project activities, especially research projects and new product development projects. Common tools in project management such as the critical path method do not have the ability to manage these types of projects. One of the successful tools in demonstrating the relationship between activities in Projects with recurring activities is design structure matrix (Steward,1981).

It is assumed the main cause of interrelation between activities is the information dependency. In addition to the number of feedbacks, the feedback length (the distance between the sequential number of the two activities in the matrix) is also considered. For example, if there is feedback from activity $i(i>2)$ to activity 1 , the cost of rework is 
far greater than the cost of rework between two consecutive activities (activities $i$ and $i+1$ ). Therefore, many researchers focus on obtaining a sequence of activities, taking into account all prerequisite relationships with the goal of minimizing feedback length.

In some projects because of the relations between the activities and the information dependency between them, if design and structural changes or even a lack of information occurs, they lead to duplication and repetitions and will cost lots of money on the project.

Considering that past studies from the concept of design matrix have addressed mathematical modeling to reduce repetitions and the number and length of feedbacks, in this study, in addition to mathematical modeling, using the concept of design structure matrix to reduce the sequential intervals of the two activities, the amount of information dependency on the two activities and the cost spent to reduce this dependence as well as the impact of the length of the feedback on the level of information dependency have been considered, and the past research of the combined scheme of these issues has been barely perceptible. So, two objectives have been defined: The first goal is to minimize the length of feedbacks (the distance between the sequential number of the two activities in the matrix), and the second is to consider the degree of information dependency $\left(\alpha_{i j}\right)$ between activities that can be determined by multiplying the activity variability (j) by the sensitivity of another activity (sensitivity of activity $i$ to j) (Ahmadi et al,2001). Reducing the degree of information dependency between activities needs cost and leads to reduction in the length of the feedback. Therefore, it is necessary to make a balance between the increase in the cost of reducing the level of dependency or information correction and reducing the length of feedback; here the second goal is defined as minimizing this increase in cost.

The background research is discussed in the next section. In the third part of the article, a two-objective model is proposed to minimize the feedback length and the costs of reducing the degree of dependence between activities. By linearizing the model to the exact solution for small and medium sized projects and the innovative solution method, an approximate solution to a large-scale model has been addressed. In the fourth section, the computational results obtained from solving the problem of the created problems are investigated and analyzed. Finally, in the fifth part, suggested solutions for future research will be presented.

\section{LITERATURE REVIEW}

Managing the development of complex products can be difficult. Because it often involves thousands of activities with complex information dependencies and finding a proper sequence of them with the goal of minimizing the length of feedback is one of the issues that has been addressed in various studies. The Design Structure Matrix (DSM) is presented as an effective tool for planning and managing product development projects through the analysis of information flow. Some researchers have used this tool to analyze complex dependencies and feedbacks. Browning (2015) addressed the literature review of the design structure matrix (Dependency structure matrix), which has become a framework for extensive modeling in many research fields. In this study, from 521 papers investigated, it has been concluded that the design structural matrix has the most prominent presence in engineering design, engineering management, system engineering, and analysis of complex systems models. To plan interdependent activities, Lin et al. (2018) used the design structural matrix to show information dependency. Based on the design structure matrix methodology and with the development of simulation-based algorithmic algorithms and developed refractive algorithms and developed genetics, this study examines the sequence of interdependent activities as to minimize the total length of feedback and reduce the completion time of the project. In the article "Reviewing the timing of the portfolio," Yassine et al. (2017) have pointed to feedback relationships that could lead to duplication and repeat, in addition to the resource constraints. 
In another approach to the product development process, Eppinger et al. (1998) evaluated the likelihood of completing this process over time. In this model, the design structure matrix has been used about the dependence of information between activities and the probability of change and the effect of repetitive activities.

Also, due to the dependence and exchange of information between activities that could affect the time of implementing the project, Silva et al. (2012) quickly transmitted information between the activities to reduce the time of the project; given the uncertainty of data and the early transmission of information, it can lead to duplication and increase the duration of the project.

Xiao et al. (2018) offered a Factored Grey Wolf Optimizer (FGWO) algorithm by adapting the convergence factor and adding weighted position factor to improve the existing Grey Wolf Optimizer (GWO). The value of convergence factor is nonlinearly changed during the iterations over the search process to better adapt the exploration and exploitation process, and the weighted position factor is linearly changed as the iteration increases to diverse the hierarchy of grey wolves.

In sequencing activities in order to minimize the total amount of feedback, Kusiak and Wang (1993) presented a triangular algorithm to identify and arrange related activities. Reza Ahmadi et al. (2001) have proposed operational frameworks for the structure of product development processes. In the proposed mathematical model, the aim of minimizing the number of repetitions between activities and finding the appropriate sequence for minimizing the overall feedback is to consider incomplete information as the design repetitions. Also, if the activity is a prerequisite for other activities that are dependent on their output, then it is said that this activity is performed under incomplete information. As a result, for a linear arrangement of activities, the total amount of incomplete information is the total weight of feedback arcs. Qian et al. (2011) used project design matrix with cross-interaction activities. The purpose of this program is to find the sequence of activities in such a way that the sum of the numbers of the uppermost diameter in the design matrix is minimized. Lin et al. (2012) in their research suggests that systems with interactive activities need to organize and plan these activities for their own success, and that the design matrix as a tool for modeling and scheduling activities with interactions. Eppinger et al. $(1994,1991,2001)$ suggested that, in addition to the number of feedbacks, the feedback length may have a dramatic effect on time and cost. In fact, feedback from the end of the process will initially give a lot more work than feedback from an activity with its previous activity. In some cases, it has been accepted that finding the right sequence with minimal overall feedback can lead to significant savings in time and cost. Recently, several mathematical models have been expanded into the feedback-length minimization problem (FLMP). For example, Meier et al. (2007) point out in their article that designing a product is important for carrying out project activities in a proper sequence; otherwise, the essential information is not available when needed, and affiliate activities will be required and when the actual information available will accelerate the flow of rework and ultimately increase the time and cost of the project. Lancaster and Cheng (2008) expanded the evolutionary algorithm and validated it by analyzing the model (criterion). Also, by measuring the improvement of the difference between successive generations, the compatibility of mutation factors in the evolutionary algorithm is examined and the design matrix is optimized using the technique used in relation to the genetic factors of the base. Qian and Lin (2014) have focused on determining the sequence of activities and minimizing the feedback length with the help of the design matrix, and developed an effective tool and innovative method for scheduling interdependent activities with the goal of minimizing the total feedback length. This issue is modeled as a linear ordering problem with quadrature objective function, which is very difficult even for small size issues. They define the degree of information dependence of activity $i$ on the activity $j,\left(O \leq \alpha_{i j} \leq 1\right)$ aij. aij can be determined from the product of the variability of activity $j$ in the sensitivity of activity $i$ relative to activity $j$ and can be estimated logically. In this paper, this topic is considered as a combination of effective feedback intervals between activities. For this reason, by providing a two-purpose mathematical model and considering the extent to which information activities are interdependent in determining the length of feedback, we seek to reduce the length of the feedback and the cost resulting from the decrease in the degree of dependence between the activities. 


\section{STATEMENT OF THE PROBLEM AND MODELING}

Consider a production development project with a set of activities $V=\{0, \ldots, n+1\}$ that represents $n$ main activity with the non-zero time and the two virtual activity 0 and $n+1$ representing the beginning and the end of the project, respectively. Among the main activities of the project, it is possible to define the prerequisite of relations, in which case the order of the activities is determined. Since some activities may require upstream or downstream information and lead feedback (backward) between activities with information dependency, the feedback length is important. Reducing information dependency, correcting, and completing information and optimizing the structure to reduce feedback length cost-time are imposed on the project, the more the reduction in dependency and information correction, the greater the cost and time spent.

Considering feedback and reducing its length as the main goal, is modeled in two ways. The first goal is to minimize feedbacks by closing the distance between the order numbers of the two activities in question, and the second goal is to minimize the cost that needs to be spent so that the degree of dependence of both feedback activities reduces it to a certain extent. Considering these two goals, if there is between the two activities of information dependency and feedback relationships, they will even be carried out within close proximity and there will be fewer activities between them, which will ultimately affect the time and cost of the project.

In the same vein, it is assumed that the number of activities of the project is limited (n activity), and the set of hard and soft prerequisite defined between the activities is certain and cannot be changed. Also, each activity has access to unlimited resources within a specified time period and, with the second goal, there is no possibility of reducing the level of information dependency to zero and eliminating dependencies, and the cost of this decrease is fixed and constant between both feedback activities.

The set of parameters, variables, and mathematical relations are defined as follows:

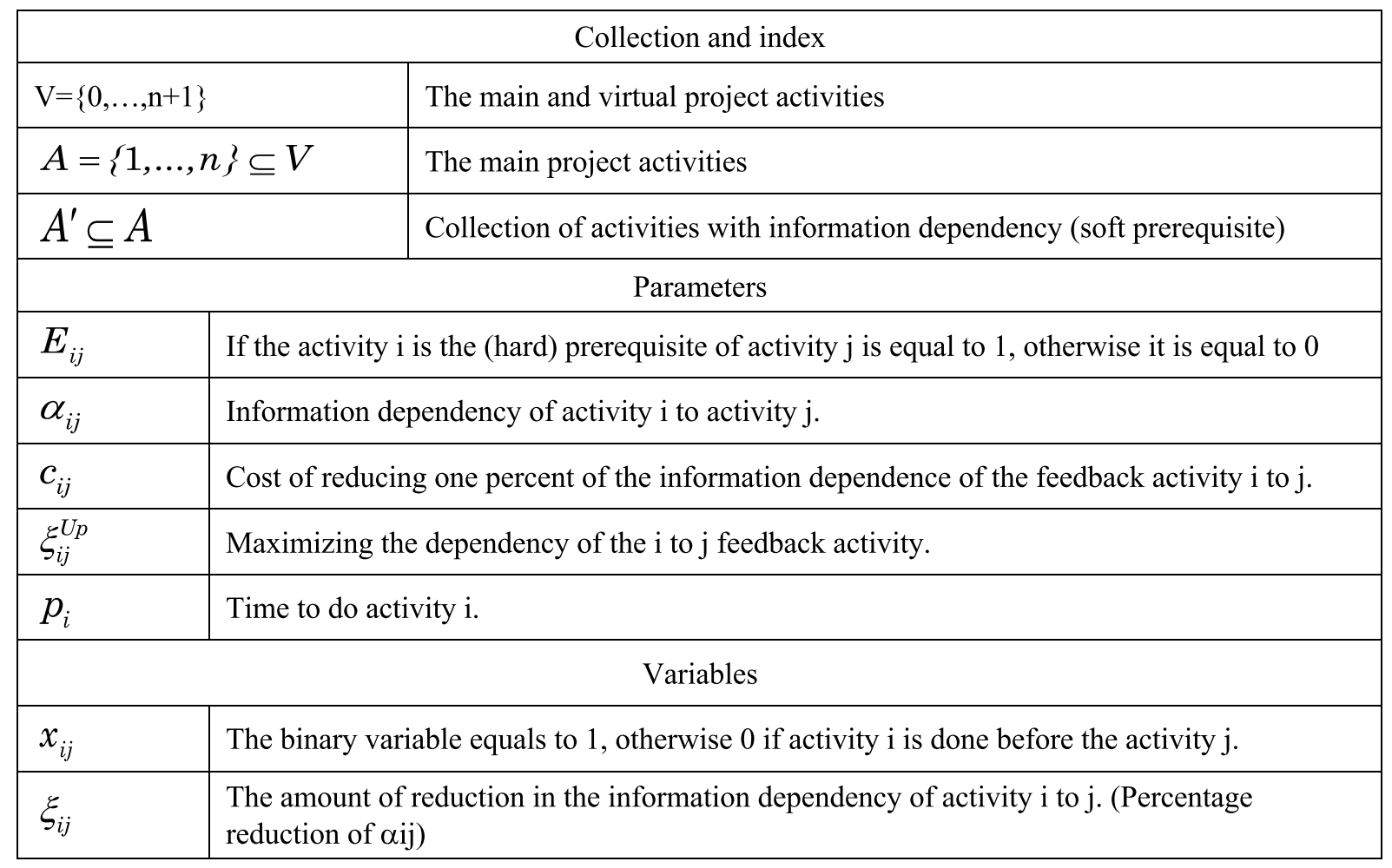




$$
\begin{aligned}
& \operatorname{Min} z_{1}=\sum_{i \in A} \sum_{j \in A}\left(1-\xi_{i j}\right) \alpha_{\mathrm{ij}} x_{i j}\left(\sum_{k \in A} x_{k j}-\sum_{k \in A} x_{k i}\right) \\
& \operatorname{Min} z_{2}=\sum_{i \in A} \sum_{j \in A} \xi_{i j} c_{\mathrm{ij}}
\end{aligned}
$$

Subject to

$$
\begin{array}{ll}
x_{i j}+x_{j i}=1 & \forall i . j \in A \\
x_{i k} \geq x_{i j}+x_{j k}-1 & \forall i . j . k \in V \\
x_{i j} \geq \mathrm{E}_{\mathrm{ij}} & \forall i . j \in V \\
O \leq \xi_{i j} \leq \xi_{\mathrm{ij}}^{\mathrm{Up}}<1 & \forall i . j \in V \\
x_{i j} \in\{0.1\} & \forall i . j \in V
\end{array}
$$

Equation (1) is the function of the first problem seeking to minimize the total feedback length (closing the distance between a sequence number) of activities with soft prerequisite. Note that the value of the objective function equals $\sum_{i \epsilon \hat{A}} \sum_{j \in \hat{A}} \alpha_{i j} x_{i j}\left(\sum_{k \epsilon A} x_{k j}-\sum_{k \epsilon A} x_{k i}\right)$ in the usual mode. If the level of information dependency between both feedback activities $i$ and $j$ is reduced to $\xi$, then the information dependency coefficient between them is equal to $\left(1-\xi_{i j}\right) \alpha_{i j}$, which is taken into account in equation (1). This relationship even reduces the number of activities performed between these two activities or reduces the amount of information to $\xi_{i j}$ between these two activities, which, if there are activities between them, does not have much feedback. Equation (2) seeks to minimize the cost of reducing the degree of information dependency of both feedback activities to the project. $\xi_{i j} c_{i j}$ is the amount of the cost that is imposed on the project due to the reduction of the $\xi_{i j}$ percent of the information dependency of activity $i$ to $\mathrm{j}$.

Equation (3) states that, for each pair of feedback activity (i.j€Á), one must take precedence over each other. Equation (4) represents the property of the violation between the priority of activities, such that if activity $i$ on activity $\mathrm{j}$, and activity $\mathrm{j}$ is preceded by activity $\mathrm{k}$, then activity $\mathrm{i}$ must be preceded by $\mathrm{k}$ activity. Limit (5) represents the prerequisite relationships between activities and ensures that if activity $i$ is the prerequisite of activity $j$ then be sure $\mathrm{i}$ activity before activity $\mathrm{j}$. Note that the virtual activity 0 to start the project is a prerequisite for all project activities so: $E_{o j}=1 \rightarrow x_{o j}=1 \quad \forall j \in V$. Also, all activities are prerequisites for $\mathrm{n}+1$ virtual activity is for the completion of the project, meaning that all project activities must be done in order to perform $\mathrm{n}+1$ virtual activity, and this virtual activity means the project is done so: $\mathrm{E}_{\mathrm{j} . \mathrm{n}+1}=1 \rightarrow x_{j . n+1}=1 \quad \forall j \epsilon V$. Equation (6) shows that the maximum reduction in the information dependency between the two activities is less than a predefined upper bound. As indicated in the modeling assumptions, there is no possibility of completely eliminating the information dependency between the two feedback activities $\mathrm{i}$ and $\mathrm{j}$, so $\xi_{\mathrm{ij}}^{\mathrm{Up}}<1$, and the amount of information dependency reduction between the two feedback activities $i$ and $j$ is between 0 and maximum $\xi_{\mathrm{ij}} \mathrm{Up}$.

From the solution of the proposed two-objective model, which is referred to in the next section, a set of Pareto's solutions is obtained, among which an answer is chosen. In the selected answer, the order of the feedback activities is determined in such a way that the feedback length is close to its lowest limit. 
We present the linearization of the proposed model based on an approximate McCormick linearization method for bilinear functions with bounded variables. Then, to solve the small and medium problems, using the precision combination of Lexicographic (Isermann,1982, Rentmeesters et al., 1996) and Augmented Epsilon Constrain (Mavrotas,2009, Aghaei et al., 2011) by the CPLEX Solver is suggested in the GAMS software, and since in the big issues the Lex-AEC combination method can after a reasonable time, be able to find the answer For the problem, we use the innovative method based on the genetic algorithm, which in either case provides the Pareto front and produces efficient solutions.

To solve the problem with the Lexicographic and Augmented Epsilon Constrain (Lex-AEC), we aim to minimize the feedback length, and the second goal is to minimize the cost spent to reduce the level of information dependence as an epsilon of constraints on the problem of applying and, Solve the following problem:

- 1: Minimize the feedback length regardless of the cost spent to reduce the level of information reliance on feedback activities.

- 2: Minimize the cost to reduce information dependency on feedback activities, regardless of the goal of minimizing feedback lengths (it is clear that the cost is 0 and cost is not reduced to reduce the dependency of information).

- 3: Calculate the PayOff matrix as follows:

$$
\mathrm{V}=\left[\begin{array}{cc}
\mathrm{FL}^{*} & \mathrm{FL}^{* \mid \operatorname{Cos} \mathrm{t}^{*}} \\
\operatorname{Cost}^{* \mid \mathrm{FL}^{*}} & \text { Cost }^{*}
\end{array}\right]
$$

- Cost*: The cost is minimized to reduce the information dependency of feedback activities, regardless of the minimum feedback length

- $\operatorname{Cost}^{* \mid \mathrm{FL}^{*}}$ : Low cost while the feedback length is in the minimum.

- FL*: Minimum feedback length

- $\mathrm{FL}^{* \mid \operatorname{Cost}^{*}}:$ Minimum feedback length in minimum cost

- 4: Calculate the radius of each target's changes as follows:

- $\operatorname{Radius}($ Cost $)=$ Cost $^{*} \mid \mathrm{FL}^{*}-$ Cost $^{*}$

- $\operatorname{Radius}(\mathrm{FL})=\mathrm{FL}^{*} \operatorname{Cost}^{*}-\mathrm{FL}^{*}$

- 5: Calculate the minimum, maximum and radius values (range of changes) for the second objective Epsilon

- $\operatorname{Min}($ Eps $)=$ Cost $^{*}$

- $\operatorname{Max}(\mathrm{Eps})=\mathrm{Cost}^{* \mid \mathrm{FL}^{*}}$

- $\mathrm{R}($ Eps $)=\operatorname{Max}($ Eps $)-\operatorname{Min}($ Eps $)$

- 6. Calculate step size for changing Epsilon: StepSize $=\mathrm{R}($ Eps $) / \mathrm{NPS}-1$

where NPS shows the number of Pareto replies.

- 7. Definition of the initial Epsilon value: Epsilon = Min(Eps)

- 8. Definition of Epsilon Constraint: $\operatorname{Cost}+$ slack $_{2}=$ Epsilon 
where Cost is the value $Z_{2}$ defined in relation (2).

- 9. Definition of the objective function of the AEC method:

- $\mathrm{z}=\mathrm{FL}+\operatorname{Radius(FL)} / \operatorname{Radius(Cost)} \times \operatorname{slack}_{2}$

where FL is the value of $Z_{1}$ defined in (13).

- 10. Run the loop for every K.

So far, the Lex-AEC hybrid approach for small- and medium-sized projects has been precisely formulated, which can get answers from the Pareto front of the problem. Increasing the dimensions of the project (number of project activities) and, as a result, increasing the number of modes determining the act of activities lead to complexity of the problem and in the relevant research, we use the meta-heuristic solving method based on the genetic algorithm to solve a problem in a larger scale to intelligently search the problem-solving space and obtain the best answer at an acceptable time.

\section{RESEARCH FINDINGS}

Following the linearization of the two-objective model and solving it with proposed methods, a series of Pareto/ungrateful solutions is obtained. The question is, which of these solutions is chosen as the best answer of the Pareto suite?

Clearly, Pareto's response (Wang et al.,2017) with the lowest feedback length cost the most and the Pareto's response with the lowest cost have the maximum feedback length. Considering the fact that cost management is an important planning elements of the project and the purpose of minimizing the length of the feedback is to minimize the cost of the project; therefore, the approach of this research to select the best answer for Pareto is that first, we select the Pareto answer which has the lowest cost, as the initial answer, and then increase the cost with a specified percentage (based on what the project can justify, for instance, $5 \%$ ), and we get the next Pareto answer with less length of feedback. We continue this process as far as the percent reduction in the length of feedback is higher than the cost increase.

In a typical example, a project with 19 main activities $A=\{1,2,3, \ldots, 19\}$ and two virtual activities with numbers 0 and 20 with limited access to resources is scheduled. In this project, the set of activities $A=\{5.6 .7 . \cdots .16\}$ has an information dependency.

The information is given in the numeric example such as the prerequisite matrix and the soft pre-matrix (information dependency), and the duration of each activity is shown in Table 1.

We also assume in this example that the maximum reduction in information dependency between feedback activities is $30 \%$ of the total information dependency $\left(\xi^{U p}{ }_{i j}=3 / 10 \alpha_{i j}\right)$. We consider the cost of reducing information dependence as 10 units of cost per unit of reduction in information dependence $\left(c_{i j}=10\right)$. To implement the Lex-AEC method, we first define the Payoff matrix, which is in the example shown in Table 4. Based on Table 4, the range of Epsilon changes in Cost + slack $_{2}=$ Epsilon is Epsilon $\in$ [0.284.65]. If the number of unanswered responses to the Lex-AEC method is equal to NOS $=10$, then the length of the step of increasing Epsilon is StepSize $=284.65-0 / 10-1=31.63$. The initial value of Epsilon is 0 and is added to each iteration of 31.63 . 
Table 1. A hard prediction matrix between project activities

(Empty or missing cells are zero, i.e., activity $\mathrm{i}$ is not a prerequisite of activity $\mathrm{j}$ )

\begin{tabular}{|c|c|c|c|c|c|c|c|c|c|c|c|c|c|c|c|c|c|c|c|}
\hline Ei.j & $\mathbf{1}$ & $\mathbf{2}$ & $\mathbf{3}$ & $\mathbf{4}$ & $\mathbf{5}$ & $\mathbf{6}$ & $\mathbf{7}$ & $\mathbf{8}$ & $\mathbf{9}$ & $\mathbf{1 0}$ & $\mathbf{1 1}$ & $\mathbf{1 2}$ & $\mathbf{1 3}$ & $\mathbf{1 4}$ & $\mathbf{1 5}$ & $\mathbf{1 6}$ & $\mathbf{1 7}$ & $\mathbf{1 8}$ & $\mathbf{1 9}$ \\
\hline $\mathbf{1}$ & & & & 1 & 1 & & & & & & & & & & & & & & \\
\hline $\mathbf{2}$ & & & & & 1 & & & & & & & & & & & & & & \\
\hline $\mathbf{3}$ & & & & & & & 1 & 1 & & & & & & & & & 1 & & \\
\hline $\mathbf{4}$ & & & & & & & & & & 1 & & & & & 1 & & 1 & & \\
\hline $\mathbf{5}$ & & & & & & & & & & & 1 & & 1 & & & & & & \\
\hline $\mathbf{6}$ & & & & & & & & 1 & & & & & & & & & & & \\
\hline $\mathbf{1 0}$ & & & & & & & & & & & & & & & & & & 1 & \\
\hline $\mathbf{1 6}$ & & & & & & & & & & & & & & & & & 1 & & \\
\hline $\mathbf{1 7}$ & & & & & & & & & & & & & & & & & & & 1 \\
\hline $\mathbf{1 8}$ & & & & & & & & & & & & & & & & & & & 1 \\
\hline
\end{tabular}

Table 2. Soft prerequisite matrix (information dependency) between project activities.

\begin{tabular}{|c|c|c|c|c|c|c|c|c|c|c|c|c|}
\hline ai.j & $\mathbf{5}$ & $\mathbf{6}$ & $\mathbf{7}$ & $\mathbf{8}$ & $\mathbf{9}$ & $\mathbf{1 0}$ & $\mathbf{1 1}$ & $\mathbf{1 2}$ & $\mathbf{1 3}$ & $\mathbf{1 4}$ & $\mathbf{1 5}$ & $\mathbf{1 6}$ \\
\hline $\mathbf{5}$ & 0 & 0 & 0 & 0 & 0 & 0 & 0.35 & 0 & 0 & 0 & 0.25 & 0 \\
\hline $\mathbf{6}$ & 0 & 0 & 0 & 0.8 & 0 & 0 & 0 & 0.55 & 0 & 0 & 0.4 & 0 \\
\hline $\mathbf{7}$ & 0.6 & 0 & 0 & 0 & 0.8 & 0.1 & 0 & 0 & 0 & 0 & 0.5 & 0 \\
\hline $\mathbf{8}$ & 0 & 0 & 0 & 0 & 0 & 0 & 0 & 0 & 0.8 & 0 & 0.2 & 0 \\
\hline $\mathbf{9}$ & 0 & 0 & 0.9 & 0 & 0 & 0 & 0.5 & 0.4 & 0 & 0 & 0.8 & 0 \\
\hline $\mathbf{1 0}$ & 0.8 & 0.5 & 0 & 0 & 0.9 & 0 & 0 & 0.8 & 0 & 0 & 0 & 0.75 \\
\hline $\mathbf{1 1}$ & 0 & 0 & 0.6 & 0 & 0 & 0 & 0 & 0 & 0 & 0 & 0 & 0 \\
\hline $\mathbf{1 2}$ & 0 & 0.85 & 0 & 0.9 & 0 & 0 & 0 & 0 & 0 & 0.35 & 0 & 0.8 \\
\hline $\mathbf{1 3}$ & 0.9 & 0 & 0 & 0 & 0.8 & 0 & 0.9 & 0 & 0 & 0 & 0 & 0 \\
\hline $\mathbf{1 4}$ & 0 & 0 & 0 & 0 & 0.5 & 0 & 0.5 & 0 & 0.9 & 0 & 0 & 0.8 \\
\hline $\mathbf{1 5}$ & 0 & 0.8 & 0 & 0.7 & 0.6 & 0.7 & 0 & 0 & 0 & 0.7 & 0 & 0.2 \\
\hline $\mathbf{1 6}$ & 0.4 & 0 & 0.7 & 0 & 0 & 0 & 0.8 & 0 & 0 & 0 & 0 & 0 \\
\hline
\end{tabular}

Table 3. The earliest, the shortest, and the duration of each project activity.

\begin{tabular}{|c|c|c|c|c|c|c|c|c|c|c|c|}
\hline activity & $\mathbf{0}$ & $\mathbf{1}$ & $\mathbf{2}$ & $\mathbf{3}$ & $\mathbf{4}$ & $\mathbf{5}$ & $\mathbf{6}$ & $\mathbf{7}$ & $\mathbf{8}$ & $\mathbf{9}$ & $\mathbf{1 0}$ \\
\hline ES(i) & 0 & 0 & 0 & 0 & 10 & 10 & 10 & 30 & 30 & 30 & 40 \\
\hline LS(i) & 0 & 100 & 100 & 200 & 200 & 400 & 400 & 300 & 300 & 500 & 600 \\
\hline P(i) & 0 & 42 & 38 & 42 & 53 & 70 & 80 & 90 & 110 & 90 & 55 \\
\hline Activity & $\mathbf{1 1}$ & $\mathbf{1 2}$ & $\mathbf{1 3}$ & $\mathbf{1 4}$ & $\mathbf{1 5}$ & $\mathbf{1 6}$ & $\mathbf{1 7}$ & $\mathbf{1 8}$ & $\mathbf{1 9}$ & $\mathbf{2 0}$ & $\mathbf{2 1}$ \\
\hline ES(i) & 50 & 70 & 70 & 80 & 80 & 80 & 90 & 100 & 100 & 100 & 0 \\
\hline LS(i) & 740 & 750 & 750 & 800 & 800 & 800 & 900 & 1000 & 1000 & 1000 & 500 \\
\hline P(i) & 60 & 70 & 100 & 25 & 40 & 70 & 120 & 80 & 115 & 0 & 0 \\
\hline
\end{tabular}


Table 4. Outcome matrix for cost reduction of information dependency and feedback length.

\begin{tabular}{|c|c|c|}
\hline & FL & Cost \\
\hline FL & 57.3 & 91.5 \\
\hline Cost & 284.65 & 0 \\
\hline
\end{tabular}

After determining the Payoff matrix, the initial value of Epsilon and Step Size of the Pareto front of the LexAEC method, or the balance between the feedback length and the cost of reducing the information dependency, is reported in table 5 .

Table 5. Pareto Front (Feedback Balance and Cost-Reduction of Information Dependence) by Lex-AEC.

\begin{tabular}{|c|c|c|c|}
\hline & FL & cost & Epsilon \\
\hline $\mathbf{1}$ & 91.5 & 0 & 0.00 \\
\hline $\mathbf{2}$ & 78.34 & 31.63 & 31.63 \\
\hline $\mathbf{3}$ & 71.78 & 63.26 & 63.26 \\
\hline $\mathbf{4}$ & 66.43 & 94.88 & 94.88 \\
\hline $\mathbf{5}$ & 63.5 & 120.12 & 126.51 \\
\hline $\mathbf{6}$ & 61.5 & 148.05 & 158.14 \\
\hline $\mathbf{7}$ & 59.8 & 189.77 & 189.77 \\
\hline $\mathbf{8}$ & 58.35 & 210.70 & 221.39 \\
\hline $\mathbf{9}$ & 57.8 & 245.90 & 253.02 \\
\hline $\mathbf{1 0}$ & 57.3 & 284.65 & 284.65 \\
\hline
\end{tabular}

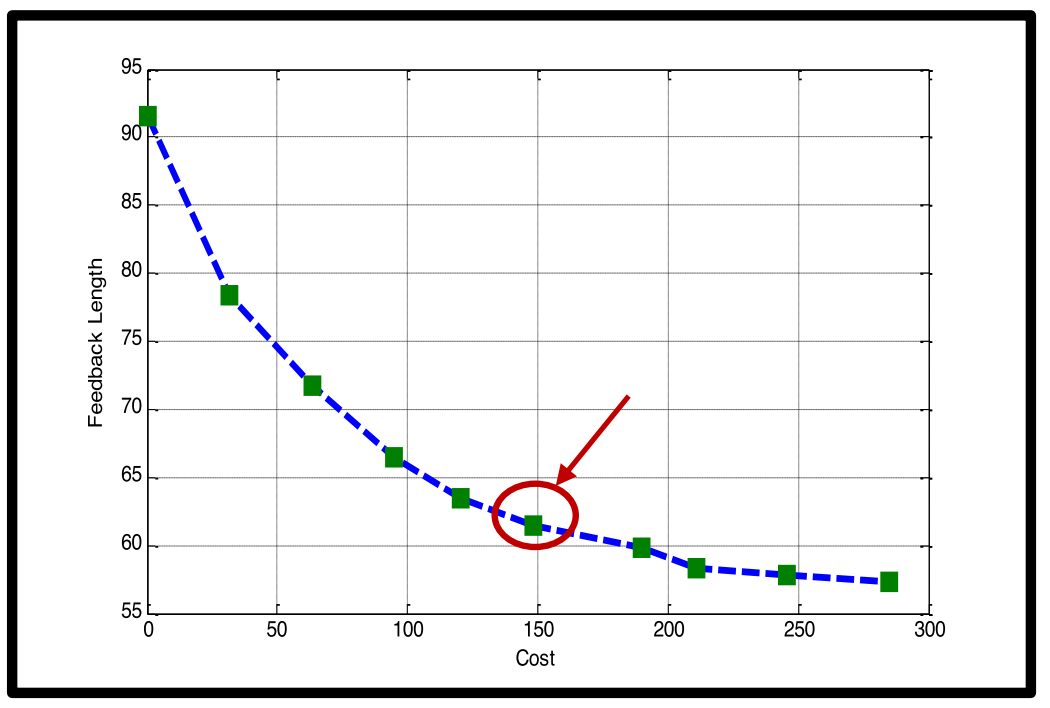

Figure 1. Pareto Front Line Charts for the feedback length and the cost of reducing the information dependency with the Lex-AEC method. 
In choosing an answer, we use the Pareto answer set, so that the cost is initially set to its lowest value (cost 0 ) and the maximum feedback length is determined, and then the cost increases to a certain amount and the feedback length is reduced. This process continues to the extent that the cost increase will be significantly reduced during feedback.

In figure 1, the plot of the Pareto front is a numerical example. Based on the proposed benchmark, the Pareto 6 response was chosen at a cost of 148.05 units and a response time of 61.5 because, after that, the cost increased by $93 \%$ (increase from 148.05 units to 284.65 units), the feedback length was not significantly reduced, and roughly $7 \%$ decrease (from 61.5 to 57.3 ).

\section{CONCLUSIONS AND SUGGESTIONS}

In this paper, we focused on the problem of minimizing the feedback length, taking into account all the tightly predicted relationships between activities and feedback that is due to information deficits or structural changes. In this regard, a two-factor mathematical model was defined. The first goal is to minimize the feedback length (the distance between the sequence number of the two activities in the matrix), and the second is to consider the degree of information dependency $\left(\alpha_{i j}\right)$ between the activities that can be multiplied by the activity variability $(\mathrm{j})$ in the other activity sensitivity (sensitivity of the actions $i$ to $j$ ) to be determined.

To solve the two-objective model, we used the Lex-AEC combination method. In the Lex-AEC method, part of the Pareto-Global solutions is obtained. The results show that paying much attention to the feedback length increases the costs of reducing the degree of dependency. While over time and with flexibility as to the feedback length, the cost of reducing the degree of dependence on the gradient is reduced to a point where the lack of attention or flexibility in the feedback length does not have a significant effect on cost reduction.

On the other hand, with the increase in the issue dimension (especially the number of project activities), the implementation time of the Lex-AEC combination method expands exponentially, which has been utilized in the corresponding repository based on the genetic algorithm-based meta-analysis solution for large-scale problem solving. Since the Pareto Frontier in the close-up approach is close to the Pareto Front in the exact solution method, and there is little difference between the answers of these two methods, it is possible to use the hyperbolic logarithm for solving large-scale projects as well.

This research can be improved in a number of areas, which can be highlighted by the following:

- Consideration of possible interruptions in activities with feedback relationships

- In the current research, we have considered the probability that the activity is not performed correctly or disrupted in any activity, so that it can be considered as a possible or fuzzy parameter in problem modeling.

- Model development for modes where each activity can be done in several ways

- In the current research, we assume that each activity can only be done in a manner and at a given time using a specific source, so that it can be defined for each activity in several different ways, which has its own time and source.

- The variability of costs caused by the decrease in the degree of dependency 
- In the current research, we assume that the cost per unit of the degree of dependence of the feedback activity is constant, while the cost can be considered for a higher percentage of the degree of dependence of the variable.

\section{REFERENCES}

Steward, D.V. 1981. The Design Structure System: A Method for Managing the Design of Complex System: IEEE Transactions on Engineering. EM-28(3): 71-74. https://ieeexplore.ieee.org/abstract/document/6448589

R,Ahmadi and A,Roemer and H,Wang. 2001. Structuring Product Development Processes: European Journal of Operational Research. 130(3):539-558. https:/www.sciencedirect.com/science/article/abs/pii/S0377221799004129

Tyson R. Browning. 2015. Design Structure Matrix Extensions and Innovations: A Survey and New Opportunities: IEEE Transactions On Engineering Management. 63(1): 27-52. https://ieeexplore.ieee.org/abstract/document/7332930/

Jun Lin, Weihao Huang, Yanjun Qian, and Xi Zhao. 2018. Scheduling Interrelated Activities Using InsertionBased Heuristics: IEEE Transactions On Engineering Management. 65(1): 113-127. https://ieeexplore.ieee.org/abstract/document/8068940

Ali A. Yassine, Omar Mostafa, Tyson R. Browning .2017. Scheduling Multiple, Resource-Constrained, Iterative, Product Development Projects with Genetic Algorithms: Computers \& Industrial Engineering. 107:39-56. https://www.sciencedirect.com/science/article/abs/pii/S0360835217300840

Carrascosa.M, Eppinger.S, Whitney.D. 1998. Using the design structure matrix to estimate product development time. ASME Design Engineering Technical Conferences. http://web.mit.edu/people/eppinger/pdf/Carrascosa_DAC_1998.pdf

Campos Silva.D., et al. 2012. Impact of Premature Information Transfer on Cost and Development Time of Projects. IEEE Transaction on Engineering Management. 59(4): 692-704. https://ieeexplore.ieee.org/abstract/document/6175943/

W.Xiao, H.Deng, Y. Sheng, and L.Hu. 2018. FACTORED GREY WOLF OPTIMIZER WITH APPLICATION TO RESOURCE-CONSTRAINED PROJECT SCHEDULING. International Journal of Innovative Computing. 14(3): 1349-4198. http://www.ijicic.org/ijicic-140308.pdf

A, Kusiak and J. Wang. 1993. Efficient organizing of design activities. Int. J. Prod. Res. 31: 753-769. https://www.tandfonline.com/doi/abs/10.1080/00207549308956755

Y.J. Qian, J. Lin, T. N. Goh, and M. Xie. 2011. A novel approach to DSM based activity sequencing problem. IEEE Trans. Eng. Manage. 58(4): 688-705. https://ieeexplore.ieee.org/abstract/document/5741721/

J. Lin, Y. J. Qian, A. A. Yassine, and W. T. Cui. 2012. A fuzzy approach for sequencing interrelated activities in a DSM. Int. J. Prod. Res. 50: 7012-7025. https://www.tandfonline.com/doi/abs/10.1080/00207543.2011.648779

S. D. Eppinger; D. E. Whitney; R. P. Smith, and D. A. Gebala. 1994. A model based method for organizing tasks in product development. Res. Eng .Design. 6: 1-13. https://link.springer.com/article/10.1007/BF01588087

D. A. Gebala and S. D. Eppinger. 1991. Methods for analyzing design procedures. presented at the ASME Int. Design Engineering Technical Conf.,Miami, FL, USA, 22-25.

https://dspace.mit.edu/bitstream/handle/1721.1/48382/methodsforanalyz00geba.pdf?sequence=1 
S. D. Eppinger. 2001. Innovation at the speed of information. Harvard Business Review. 79(1):149-15. https://www.fast-bridge.net/wpcontent/uploads/resources/Innnovation\%20at\%20the\%20speed\%20of\%20information.pdf

C. Meier, A. A. Yassine, and T. R. Browning. 2007. Design process sequencing with competent genetic algorithms. J. Mech. Design. 129(6): 566-585. https://asmedigitalcollection.asme.org/mechanicaldesign/article-abstract/129/6/566/451363

J. Lancaster and K. Cheng. 2008. A fitness differential adaptive parameter controlled evolutionary algorithm with application to the design structure matrix. Int. J. Prod. Res 46(18): 5043-5057. https://www.tandfonline.com/doi/abs/10.1080/00207540701324176

Y. Qian and J. Lin. 2014. Organizing Interrelated Activities in Complex Product Development. Engineering Management, IEEE Transactions on, 61(2):298-309. https://ieeexplore.ieee.org/abstract/document/6650069

Isermann, H. 1982. Linear lexicographic optimization. OR Spectrum 4(4):223-22. https://link.springer.com/article/10.1007/BF01782758

Rentmeesters, M. J., W. K. Tsai and K.-J. Lin. 1996. A theory of lexicographic multi-criteria optimization. In 2nd IEEE International Conference on Engineering of Complex Computer Systems, Oct 21-25. https://ieeexplore.ieee.org/abstract/document/558386

Mavrotas, G. 2009. Effective implementation of the $\varepsilon$-constraint method in multi-objective mathematical programming problems. Applied mathematics and computation 213(2):455-465. https://www.sciencedirect.com/science/article/abs/pii/S0096300309002574

Aghaei, J., N. Amjady and H. A. Shayanfar. 2011. Multiobjective electricity market clearing considering dynamic security by lexicographic optimization and augmented epsilon constraint method. Applied Soft Computing 11(4):3846-3858. https://www.sciencedirect.com/science/article/abs/pii/S1568494611000822

N.Wang, W-J.Zhao, N.Wu, D.Wu. 2017. Multi-objective optimization: a method for selecting the optimal solution from Pareto non-inferior solutions. Expert Systems With Application. 74: 96-104. https://www.sciencedirect.com/science/article/abs/pii/S0957417417300040 